\title{
Comparative study of tibial (single) and tibiofemoral (double) osteotomy for osteoarthrosis and rheumatoid arthritis
}

\author{
J. M. I. IVESON, E. B. LONGTON, AND V. WRIGHT \\ From the Rheumatism Research Unit, University of Leeds, and the General Infirmary, Leeds
}

SUMMARY Osteotomies on 101 knees in 79 patients were assessed either prospectively or retrospectively. High tibial osteotomy was performed in 54 knees (27 with rheumatoid arthritis (RA) and 27 with osteoarthrosis (OA)) and double (tibiofemoral) osteotomy in 47 knees (25 RA and $22 \mathrm{OA}$ ), and were assessed prospectively in 46 and retrospectively in 55 . Using a subjective assessment, $65 \%$ showed some improvement $-70 \%$ of the single and $60 \%$ of the double osteotomies. Of the four groups (OA single or double, RA single or double), OA knees having a single osteotomy improved most frequently $(74 \%)$, and OA knees having a double osteotomy least frequently $(50 \%)$. Significant improvements in pain score and angular deformity were recorded. The mean range of movement of the operated knee was significantly reduced, and was particularly evident in those knees having a double osteotomy. We conclude that double osteotomies tend to have a higher incidence of complications, including impaired movement, and are not more efficient in relieving pain than single osteotomies in either OA or RA.

Since tibial osteotomy was introduced by Jackson in 1958 into the management of osteoarthrosis (OA) of the knee it has been established as a useful procedure in the relief of pain and angular deformity resulting from this disorder (Wardle, 1964; Veenemans, 1962; Dawson, 1965; Gunn, 1966; Wiley, 1967; Cauchoix et al., 1968; Ahlberg et al., 1968; Bauer et al., 1969; Devas, 1969; Harris and Kostnik, 1970; Shoji and Insall, 1973; Coventry, 1973; Insall et al., 1974; Jackson and Waugh, 1974) and to a lesser extent in rheumatoid arthritis (RA) (Gunn, 1966; Devas, 1969; Coventry, 1973; Jackson and Waugh, 1974), although Ahlberg et al. (1968) obtained poor results in RA. In 1969 Benjamin described a combined tibial and femoral double osteotomy, which has also been reported to give good results in both $\mathrm{OA}$ and RA of the knee (Benjamin, 1969; Huskisson and Phillips, 1973; Angel et al., 1974), often in the absence of deformity. To investigate whether one operation has any advantage over the other, a mixed prospective and retrospective study of these two procedures was conducted in both OA and RA.

Accepted for publication October 8, 1976

Correspondence to Dr. J. M. I. Iveson, York District Hospital, Wigginton Road, York YO3 7HE.

\section{Material and methods}

The indications for operation were (1) severe pain unrelieved by lengthy conservative treatment, with or without (2) angular deformity of the knee. Marked degrees of fixed flexion deformity or instability were considered unsuitable and such patients were excluded. Lesser degrees of flexion deformity were corrected preoperatively by serial splinting in some cases. Patients seen prospectively were randomly assigned to have either a single or double osteotomy.

\section{SURGICAL TECHNIQUE}

Standard high tibial and tibiofemoral (double) osteotomies, the latter after the technique of Benjamin (1969), as shown to one of us (E.B.L.), were performed by the same surgeon (E.B.L.). The following points of detail and classification should, however, be made.

(1) High tibial osteotomy was extra-articular but made proximal to the tibial tubercle and superior tibiofibular joint. The fibula was not divided. A pneumatic tourniquet was used after the limb had been exsanguinated by means of an Esmarch rubber bandage, except in those cases where the skin appeared vulnerable, when it was achieved by raising 
the limb. The skin incision was longitudinal, and slightly oblique, running distally for $7 \cdot 5-10 \mathrm{~cm}$ from just proximal to the joint line. The osteotomes were guided by vision and touch. The knee was flexed to $90^{\circ}$ before dividing the posterior cortex. A small pressure bandage was put in place before releasing the tourniquet and application of the plaster of Paris cylinder.

(2) Tibiofemoral osteotomy. Both tibial and femoral osteotomies were made through the joint, wide access being gained through a long anteromedial incision. Femoral osteotomy involved transecting the patellar articular surface in order to cut the femoral condyles close to the joint line. The use of a bulky compression bandage before plastering was avoided by the release of the pneumatic tourniquet before wound closure, thus allowing control of the ensuing haemorrhage before skin closure. The escape of blood was facilitated by an extremely loose closure of the synovium and capsule. Usually only two catgut stitches were placed through the superomedial and inferomedial areas of the wound edges where larger blood vessels are often evident. Skin closure was with a continuous nylon stitch.

(3) In single or double osteotomies a wedge of bone with either a medial or lateral base was removed from the tibia in those cases requiring correction of an angular deformity; that is, a medially based wedge to correct a valgus deformity, and a laterally based wedge to correct a varus deformity. There was no particular technical difficulty in performing either form of osteotomy, as the wide exposure of the joint involved in the double osteotomy renders the upper tibia easier of access for division. There was therefore no significant difference in tourniquet time (15-20 minutes) in the two operations because the circulation was restored in the double osteotomies before wound closure. This obviated the necessity for a bulky pressure bandage, which with a superadded plaster of Paris splint, would create a very cumbersome dressing and cause early walking to be difficult.

Weight bearing and walking were encouraged within a week, even in cases of bilateral operations, and the patient was discharged home at approximately the tenth day or to a convalescent hospital if other joint involvement made independent living impossible. Plaster and sutures were removed at the fifth week in the absence of obvious complications and a course of physiotherapy was started, which continued in outpatients. Radiographs were not helpful in confirming union at the osteotomy site because of the close compression of the bone in juxta-articular osteotomies especially after early weight bearing. Manipulation under an anaesthetic was not performed routinely but was reserved for those patients considered by the surgeon to be regaining the range of movement too slowly incompletely.

\section{ASSESSMENTS}

These were performed by one observer (J.M.I.I.) ิㅡㅁ all occasions. The prospective patients were seefi before operation and at 3- to 6-monthly interva然 from the time of operation. Approximately half of the retrospective patients were seen on more than one occasion postoperatively. All objective asses?ments were made with the patient lying on an examination couch.

The following assessments were made. (a) Fun tional status 1-5 (based on that used by the Empiess Rheumatism Council's Multicentre Gold Tria9 1960). (b) Functional assessment by an occupational therapist. This was a subjective test-the patien answered specific questions relating to everyday actions. (c) Pain was classified by the patient as absent, mild, moderate, or severe (0-3) for each of three situations-walking, resting, and in bed. maximum combined pain score of 9 was possible (d) The range of movement was measured using 8 Zimmer orthopaedic goniometer. A fully extended knee was recorded as $0^{\circ}$, and full flexion was possible through an arc of approximately $140^{\circ}$ in a normal knee. Extension (quadriceps) lag was measured whe the unsupported leg was raised from the couc (e) The tibiofemoral angle was measured with the patient lying on a couch. An angle of $170-180^{\circ}$ w considered to be within the normal range. Less tha $170^{\circ}$ was classified as a valgus deformity an greater than $180^{\circ}$ a varus deformity. (f) Instabilie or laxity of the knee joint in a mediolateral plane was detected by the standard clinical method of rocking the knee, with minimal force and in full (of almost full) extension. The same rocking motio was induced in the anteroposterior plane with the knee flexed to $90^{\circ}$. The result was recorded as nil to severe (0-3). A similar scale was used for $(\mathrm{g})$ tendetness (squeeze pressure) in a mediolateral plane, (h) swelling (soft tissue only), and (i) warmt (j) The opinion of both patient and observer on the operation result was recorded as 'excellent', 'goodect 'fair', 'no change', or 'worse'.

Both operated and unoperated limbs wens assessed. The $\chi^{2}$ test was used for analysis of the numbers of patients showing improvement of deterioration, and Student's $t$ test for measurements of movement and angular deformity. Pain scores were analysed by the Mann-Whitney $U$ test.

\section{PATIENTS STUDIED}

General

Osteotomies on 101 knees in 79 patients $(19$ male 60 female) were assessed. Bilateral operations were 
performed in 22 patients (i.e. 44 knees)-on the same day in 12 patients, and after a mean interval of 9.2 months in the remainder. The mean age was $63 \cdot 1$ years.

\section{$O A$}

Forty-nine osteoarthrotic knees underwent osteotomy-27 tibial and 22 double. Operations were assessed prospectively in 21 (11 double, 10 single) and retrospectively in 28 (Fig. 1). OA had been present for a mean of 16.2 years and the mean age of the patients was 59.7 years.

\section{$R A$}

Fifty-two rheumatoid knees were included-27 tibial and 25 double osteotomies. The operations were assessed prospectively in 25 knees. The mean age of the patients was 66 years and the disease had been present for a mean of 15 years.

\section{Results}

\section{SUBJeCtive ASSESSMENTS (Figs. 2, 3)}

\section{(1) Patient opinion}

(a) General. Improvement (excellent, good, or fair) was noted in $66(65 \%)$ of the 101 knees. In $70 \%$ of the single and $60 \%$ of the double osteotomies improvement was noted, and excellent or good results were recorded in $50 \%$ of the single and $43 \%$ of the double osteotomies (the difference between single and double is not significant). The proportion of prospective and retrospective patients improving was similar (62\% and $67 \%$ respectively). The operation resulted in worsening of the knee in 8 of the 101 knees $(7.9 \%) .5$ of these had had double osteotomies (Fig. 2).

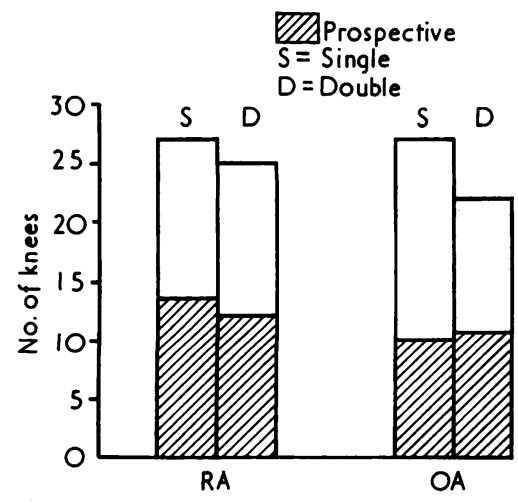

Fig. 1 The ratio of prospective and retrospective assessment of 101 knees. $R A=$ rheumatoid arthritis; $O A=$ osteoarthrosis.

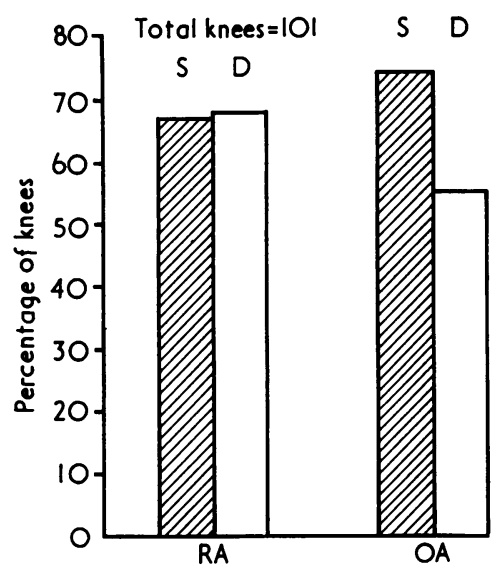

Fig. 2 Patient's assessment of the operative resultimproved.

(b) $O A$. Improvement was reported in $63.3 \%$ of the knees with excellent results in $51 \%$. Single osteotomies improved the knee more frequently $(74 \%)$ than double osteotomies $(55 \%)$ but the difference was not statistically significant. Good or excellent results occurred in $67 \%$ of the single and $36 \%$ of the double osteotomies $(0.1>\mathrm{P}>0.05)$ (Fig. 3).

Five knees were made worse as a result of the double osteotomy. In three this was due to poor postoperative function with marked impairment of movement. In one patient with parkinsonism the femoral element of the osteotomy failed to unite; in another severe bleeding postoperatively resulted in a haemarthrosis and a stiff knee. One knee was made worse by tibial osteotomy.

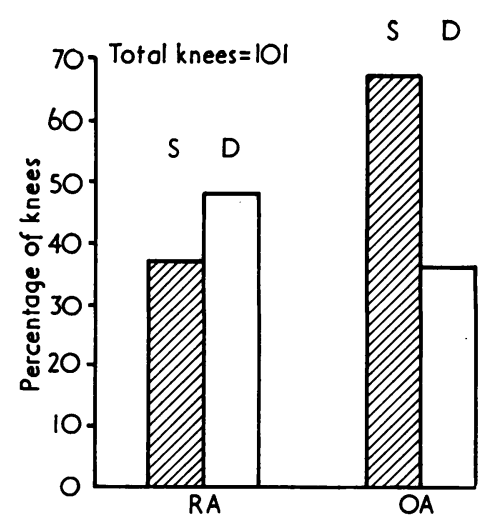

Fig. 3 Patient's assessment of the operation resultexcellent or good. 
(c) $R A$. Improvement was reported in $67 \%$ of single and $68 \%$ of the double osteotomies. This was 'good' or 'excellent' in $37 \%$ of the single and $48 \%$ of the double osteotomies. One knee in each group was made worse by the operation, due to gross impairment of movement in a tibial osteotomy and a pyoarthrosis in the case of the double osteotomy.

The functional grade assigned by the physician in accordance with the grading used by the Empire Rheumatism Council's Multicentre Gold Trial (1960) was a poor index of improvement. No change occurred in $65 \%$ of the 51 in whom it was recorded both before and after operation. In only 4 was there more than one grade change, the remaining 14 $(27 \cdot 5 \%)$ changing by one grade only.

\section{OBJECTIVE ASSESSMENTS}

Comparisons were made where appropriate with the unoperated knee. The separate consideration of retrospective and prospective series had no significant effect on the results. In many of the parameters (e.g. pain score, range of movement) the results were of necessity prospective as little retrospective information was available.

\section{(2) Pain score}

(a) General. Whereas none of the preoperative knees classified for pain were entirely pain-free, $31 \%$ of the postoperative were. Severe pain was recorded in $34 \%$ of the knees preoperatively and $3 \%$ postoperatively. This is in marked contrast to the unoperated knees, with the same proportion of knees pain-free both before and after surgery (Fig. 4). There was a markedly significant difference $(P<$ 0.001 ) between pre- and postoperative mean pain scores for all four operative groups. The pain relief afforded by either single or double osteotomy was not significantly different.

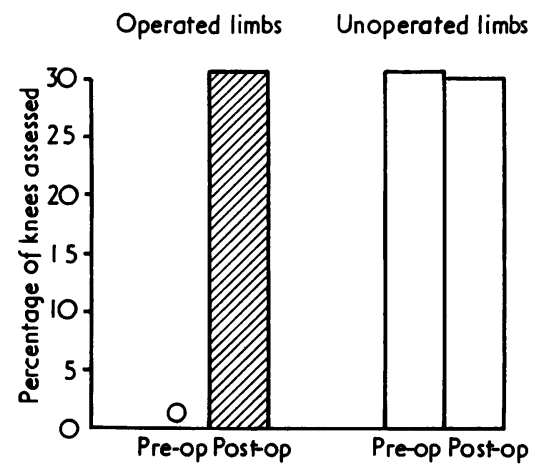

Fig 4. Proportion of all knees, before and after osteotomy, which were free of pain.

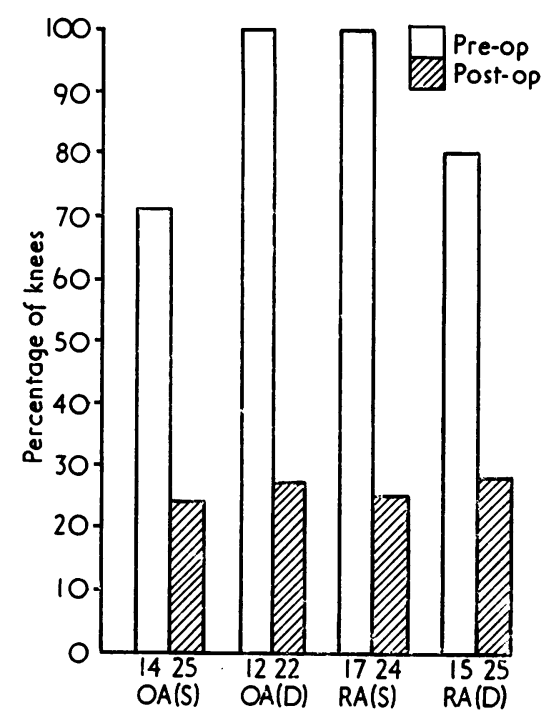

Fig. 5 A comparison of the percentage of knees, befor and after osteotomy, with moderate to severe pain.

(b) $O A$ (Fig. 5). No knee was pain-free preoperatively After single osteotomy $40 \%$ of the knees were pain-free, in contrast to $18 \%$ of those undergoigg double osteotomy. Preoperatively, moderate severe pain was recorded in $71 \%$ of those knees undergoing tibial osteotomy and $100 \%$ of the double osteotomies. After operation these figures were reduced to $24 \%$ and $27 \%$ respectively. More that half of both surgical groups improved by two more of the possible four pain groups.

(c) $R A$ (Fig. 5). Again, no knee was entirely pain-fre preoperatively, but $100 \%$ of those knees undergoi tibial and $80 \%$ of the double osteotomies had moderate to severe pain. The figures postoperatively were $25 \%$ and $28 \%$ respectively.

\section{(3) Range of movement (Fig. 6)}

The preoperative mean range of movement was greater than $90^{\circ}$ in all groups except OA knees subsequently having a single osteotomy. Postoperatively all groups had a mean range of moveme of less than $90^{\circ}$, the operation significantly reducias the mean $( \pm \mathrm{SD})$ range of the $101 \mathrm{knees}$ from $99^{\circ}$ $( \pm 29)$ to $83^{\circ}( \pm 28)$. In contrast the unoperated limb retained its range, and exhibited a greater initial range of $110^{\circ}( \pm 27)$. The loss of movement was particularly noticeable in both double osteotong groups $(\mathrm{P}<0.05)$, especially in osteoarthrotic knees.

(a) $O A$. A significant reduction in the range occurre in the double osteotomy group from $102^{\circ}( \pm 19)$ to $82^{\circ}( \pm 2)$. 


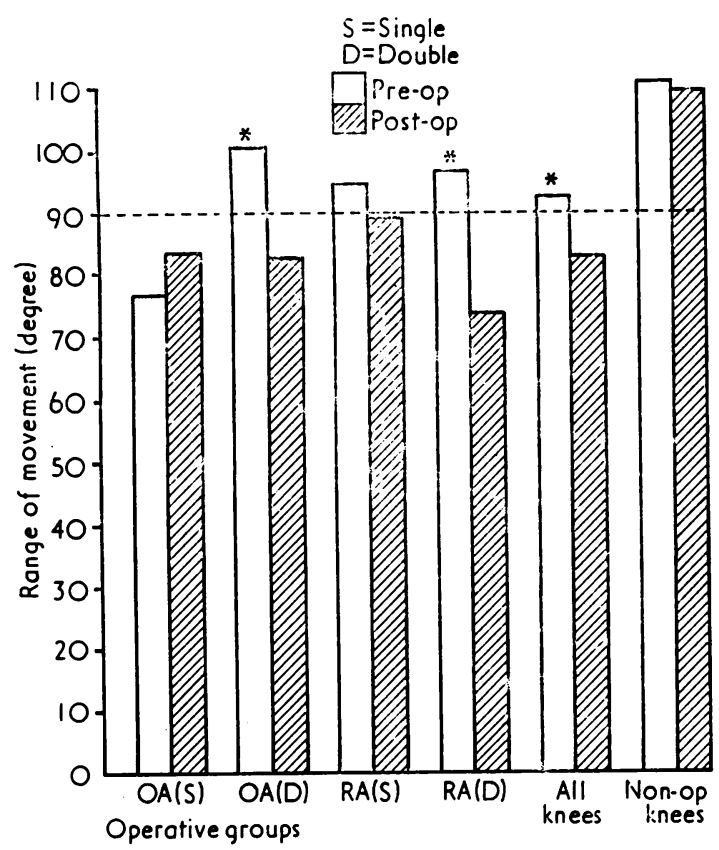

Fig. 6 Change in range of movement after knee osteotomy compared with the unoperated knee $\left({ }^{*} P<0.05\right.$, significantly different $)$.

(b) $R A$. A reduction in the mean range of movement occurred in both groups but was significant only in the double osteotomy group, when an initial range of $96^{\circ}( \pm 16)$ fell to $75^{\circ}( \pm 34)$.

\section{(4) Angular deformity}

Varus deformity was mainly caused by OA, and conversely valgus deformity was confined to the rheumatoid groups.

(a) $O A$. The mean preoperative tibiofemoral angle for the single osteotomy group was $181^{\circ}( \pm 5)$ and, for the double osteotomy group $189^{\circ}( \pm 8)$. Three of the eight knees in the single osteotomy group, in whom the angle was measured preoperatively were in varus, as were ten of the eleven measured double osteotomies. Two of the three single osteotomies and seven of the ten double osteotomies with initial varus were successful. Two of five knees which had normal tibiofemoral angles preoperatively which underwent single osteotomies had successful osteotomies.

(b) $R A$. The mean preoperative tibiofemoral angle was $171^{\circ}( \pm 9)$ for the single osteotomy group, and $169^{\circ}( \pm 10)$ for the double osteotomy group. Preoperative valgus deformity (i.e. $>10^{\circ}$ from the vertical) was present in 5 of the 15 knees measured preoperatively in the single osteotomy group (mean valgus $19^{\circ}$ ) and in seven of the twelve knees in the double osteotomy group (mean valgus $18^{\circ}$ ). Varus deformity was present in two in the single and one of the double osteotomy groups. Of the valgus knees, three in the single group and five in the double osteotomy group were adjudged successful results. Seven of eight knees, with normal tibiofemoral angles preoperatively, which underwent single osteotomies and three of four knees, undergoing double osteotomies, benefited from the surgery.

Although the numbers are insufficient to draw firm conclusions about the effect of preoperative angular deformity on the outcome of operation in each group, the impression is given by the combined figures that the outcome of the osteotomy is not affected by the presence or absence of an angular deformity and that a 'straight' knee preoperatively may respond satisfactorily. (Fig. 7.)

\section{(5) Instability}

This variable was assessed prospectively in 44 knees but there were only a small number in each group (Fig. 8). Approximately $40 \%$ of all knees were stable preoperatively and only $14 \%$ exhibited moderate to severe instability. There was no significant change in the grouping after operation, although all knees tended to be more stable. The unoperated limbs had significantly less instability before operation $(\mathrm{P}<$ 0.05 ), which also remained unchanged at the time of operation.

Postoperative instability was associated with a significant reduction in the number of good results $(P<0.005)$. Five of six preoperative knees and 6

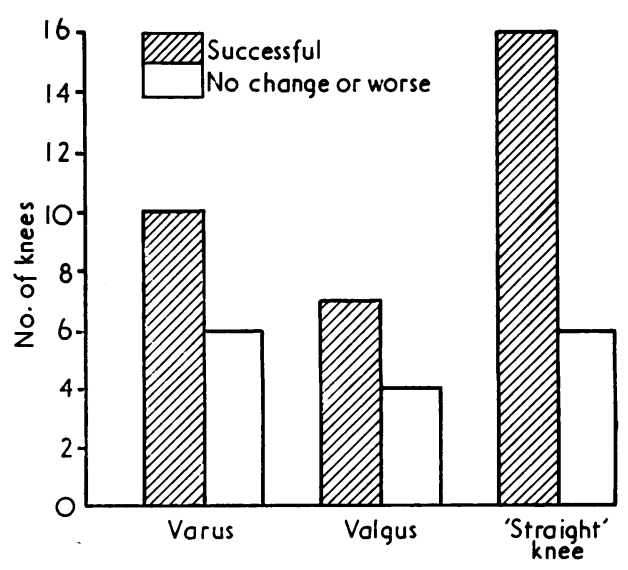

Fig. 7 The result of osteotomy (either single or double) in knees with and without angular deformity before operation. 


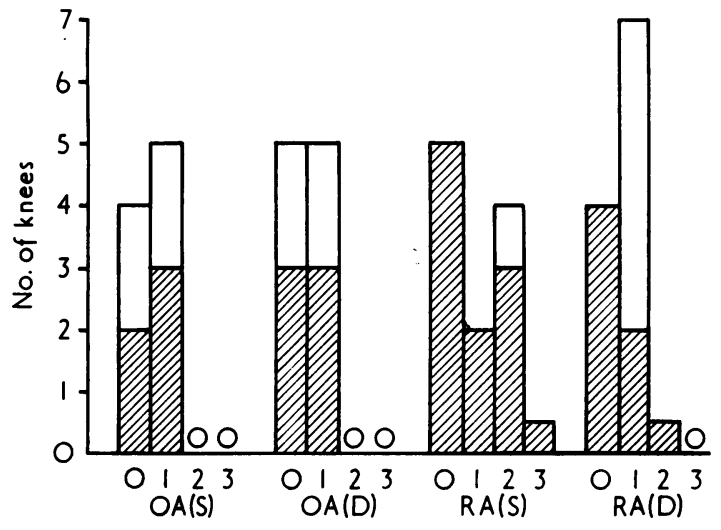

Fig. 8 Grading for instability before operation in each operative group is related to the operative results. $0-3=$ nil to severe instability. Hatched area $=$ successful operation.

$(46 \%)$ of 13 postoperative knees with moderate to severe instability were judged by the patients to have improved.

\section{COMPLICATIONS}

(a) Manipulation was not performed routinely but was reserved for those knees considered by the surgeon to have inadequate movement or no response to rehabilitation. Double osteotomies required this manoeuvre more frequently than single osteotomies, particularly in RA patients, although the difference was not significant. As a result, just over half improved $(60 \%)$ but 4 were made worse. (b) Further surgery has been performed in 2 patients with unsatisfactory results-both have had total knee replacements with the Leeds arthroplasty.

\section{Discussion}

The uncertainty about the place of double osteotomy and its value compared with single (tibial) osteotom stimulated this study. One part of the investigatio was controlled and is the only study of these operations in which patients have been randomlo allocated to one of the two procedures. It had the additional advantages that all the assessments were done by a physician independent of the surgeon and the fact that the same physician made the measures. ments throughout eliminated interobserver error.

Osteotomy produced subjective improvement iq two-thirds of the knees, confirming the value of thif procedure in arthritis of the knee. This frequency of improvement is somewhat lower than in severat previous studies (Table 1), in which complete of partial pain relief has been claimed in $63-96 \%$ of knees. Most of these reports have been retrospective and have involved smaller numbers of knees.

In the present study single osteotomies improve slightly more frequently $(70.4 \%)$ than double osteotomies (59.6\%) but osteoarthrotic and rheuma@ toid knees improved equally, even when using the same methods for assessment. The rheumatoid knees, however, especially those undergoing a double osteotomy had fewer subjectively excellent or goof results than the OA knees. Of the four groups (OAN single or double, RA single or double), OA knees having a single osteotomy had the highest incidence of improvement $(74 \%)$ and the OA doubles osteotomies the lowest $(55 \%)$. This difference was reflected in the changes in pain scores and alse objectively in the large deterioration in the meand range of movement in the $\mathrm{OA}$ double osteotomy group and a slight improvement in the OA single osteotomy group. Likewise, double osteotomies

Table 1 Reports of high tibial and tibiofemoral (double) osteotomies in which more than 40 knees have been observed

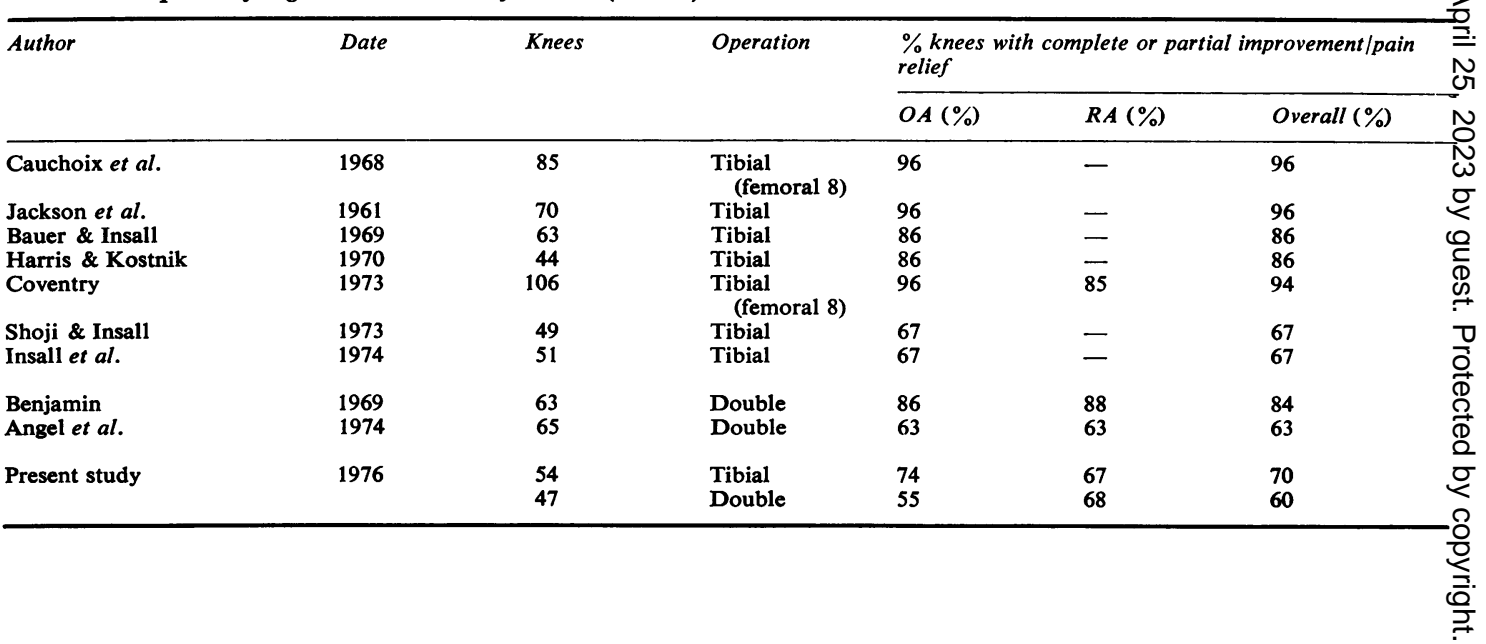


tended to require manipulation more frequently because of the slow progress in rehabilitation. Benjamin (1969) and Angel et al. (1974) achieved equally good pain relief in both OA and RA knees using a double osteotomy - a finding reflected in this study, although here the double osteotomies tended to do less well than single osteotomies. Other authors, notably Coventry (1973), feel that less assurance for pain relief, using a high tibial osteotomy, can be given in rheumatoid knees. Rheumatoid knees have been included in other studies but the numbers have been small and the response of these knees to surgery has not been separately recorded (Gunn, 1966; Ahlberg et al., 1968; Jackson and Waugh, 1974).

\section{RANGE OF MOVEMENT}

Significant reduction in the mean range of movement occurred only in the double osteotomy groups. Although OA doubles did particularly badly in this respect the result was not significantly different from the rheumatoid group. This tendency may be due to the inclusion in the study of several joints with marked varus deformity, most of which had OA as the underlying joir:t disorder and were treated with a double osteotomy introducing an unintentional bias. Both Benjamin (1969) and Angel et al. (1974) noted some reduction in the range of movement resulting from double osteotomies, the loss being greater in the OA group in the latter report. Coventry $(1965,1973)$ and Harris and Kostnik (1970) did not find any postoperative reduction in movement when using Coventry's modification of the high tibial osteotomy. These changes, the poorer results, and the more frequent complications in the double osteotomy group are presumably due to the femoral element in the surgery and the wide exposure of the joint. A higher rate of complications has been reported by several authors (Veenemans,

\section{Table 2 Complications of tibial and tibiofemoral} osteotomy

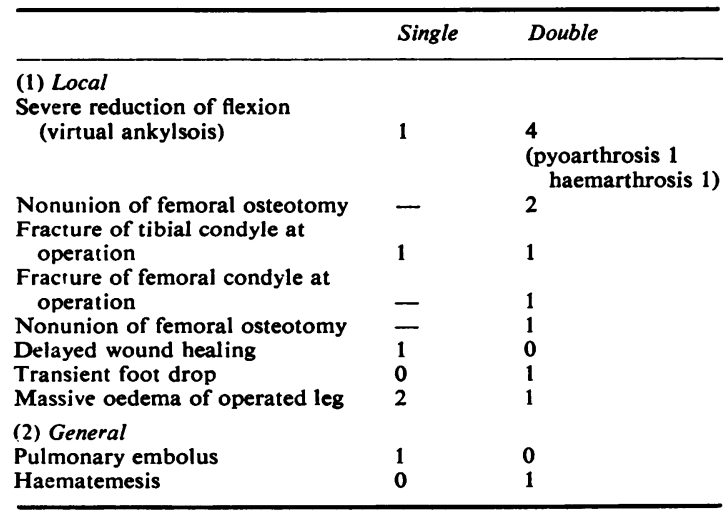

1962; Torgerson, 1965; Ahlberg et al., 1968; Bauer et al., 1969) for femoral osteotomies compared with tibial osteotomies. The necessity of opening the joint in the former is the most probable cause for this.

\section{ANG ULAR DEFORMITY}

Both undeformed knees and those with angular deformity (varus or valgus) were accepted for osteotomy. Varus deformity was mainly seen in osteoarthrotic knees and valgus deformity in rheumatoid knees. A valgus angle of less than $10^{\circ}$ was accepted as within the normal range (Torgerson, 1965; Angel et al., 1974). Neither preoperative nor postoperative angular deformity appears to be associated with subjective improvement any more frequently than a straight knee. This finding supports those of both Benjamin (1969) and Angel et al. (1974) who were able to obtain good results in the absence of angular deformity using a double osteotomy. Cauchoix et al. (1968), although preferring to do osteotomies in unicompartmental disease felt that equally satisfactory results may be obtained with bicompartmental disease-it is this latter situation which predominates in RA. In contrast, Harris and Kostnik (1970) consider high tibial osteotomy to be of value in OA knees only when there is predominant involvement of one compartment especially with angular deformity. This is supported by Coventry (1973) who stated that a knee in balance will not benefit by tibial osteotomy. Insall et al. (1974) obtained a greater proportion of improvement using a high tibial osteotomy for $\mathbf{O A}$ in varus deformity rather than valgus (Shoji and Insall, 1973). These latter results appear not to have been compared with osteotomies on straight knees.

\section{INSTA BILITY}

Severely unstable knees were usually excluded from our study as they were considered to be unsuitable for osteotomy, as has been suggested by others (Huskisson and Phillips, 1973). Occasionally, however, knees with moderate degree and, in one case, a severe degree, of instability underwent surgery. The poorer subjective outcome compared with stable knees tends to strengthen the natural desire to avoid osteotomy in unstable knees; but the results were not invariably poor and some had useful improvement in both stability and pain relief. Coventry (1973) also feels that some instability is not a contraindication to osteotomy, but he agrees with Jackson and Waugh (1961) in wishing to achieve a stable knee with correct realignment postoperatively.

Since undeformed knees may benefit from 
osteotomy it suggests that purely mechanical factors (correction of misalignment or instability) are not always the major cause of pain relief that has been suggested by some authors (Jackson and Waugh, 1961; Bauer et al., 1969; Shoji and Insall, 1973; Insall et al., 1974) but that vascular changes as suggested by Trueta (1962) and Wardle (1964) and possibly division of nerves (Trueta, 1963) may contribute. Recent experimental work by Bentley (1974) in which a nondisplacement femoral osteotomy was shown to influence favourably the healing of a papain-induced osteoarthrosis of the hip of a rabbit confirms both the efficacy of osteotomy and the vascular nature of the mechanism. As a result of this procedure increased numbers of arterioles were detected in the femoral heads and acetabula with intense marrow activity with formation of tufts of fibrocartilage on the articular surfaces.

The results of this study confirm that knees affected by both OA and RA may be successfully treated by osteotomy. Although there was no statistically significant difference between the improvement afforded by either a single or a double osteotomy in either the OA or the RA groups, it is clear that double osteotomy confers no particular advantage and may be detrimental to knee movement. The double osteotomy is no more efficient in relieving pain and is more prone to complications, and consequently the tibial osteotomy is to be preferred for most knees with either disease process. The present largely prospective study is in almost complete accord with the opinions expressed in a recent editorial (British Medical Journal, 1975), in which caution was advised against the indiscriminate use of double osteotomy, which incurs a risk of increased knee stiffness as judged by the retrospective studies available at that time.

We have pleasure in acknowledging the assistance of Mrs. J. Heasman who performed the osteotomy assessments.

\section{References}

Ahlberg, A., Scham, S., and Unander-Scharin, L. (1968). Osteotomy in degenerative and rheumatoid arthritis of the knee joint. Acta Orthopaedica Scandinavica, 39, 379-388.

Angel, J. C., Liyanage, S. P., and Griffiths, W. E. G. (1974). Double osteotomy for the relief of pain in arthritis of the knee. Rheumatology and Rehabilitation, 13, 109-119.

Bauer, G. C. H., Insall, J., and Koshino, T. (1969): Tibial osteotomy in gonarthrosis (osteoarthritis of the knee). Journal of Bone and Joint Surgery, 51A, 1545-1563.
Benjamin, A. (1969). Double osteotomy for knee pain i rheumatoid arthritis and osteoarthritis. Journal of Bone and Joint Surgery, 51B, 694-699.

Bentley, G. (1974). Symposium on Normal and Osteoarthrotic Cartilage, p. 259. Ed. by S. Y. Ali, M. W. Elves, an D. H. Leaback. Institute of Orthopaedics, London.

British Medical Journal (1975). (Editorial.) Osteotomy fợ arthritis of the knee, $1,2$.

Cauchoix, J., Duparc, J., Lemoine, A., and Deburge, (1968). L'ostéotomie dans les gonarthroses avec déviatiợ angulaire dans le plan frontal. Revue de Chirurgie Orthopédique et Réparatrice de l'Appareil Moteur, 54, 343-36\%.

Coventry, M. B. (1965). Osteotomy of the upper portion of the tibia for degenerative arthritis of the knee-a prelimw inary report. Journal of Bone and Joint Surgery, 47A 984-990.

Coventry, M. B. (1973). Osteotomy about the knee for degenerative and rheumatoid arthritis. Journal of Bone and Joint Surgery, 55A, 23-48.

Dawson, R. H. (1965). Osteotomy of the tibia for osteo arthritis of the knee. Journal of Bone and Joint Surger $\overrightarrow{0}$ 47B, 599.

Devas, M. B. (1969). High tibial osteotomy for arthritis of the knee-a method specially suitable for the elderly. Journal of Bone and Joint Surgery, 51B, 95-99.

Empire Rheumatism Council (1960). Gold therapy rheumatoid arthritis. Report of a multicentre controlle $\Phi$ trial. Annals of the Rheumatic Diseases, 19, 95-119.

Gunn, A. L. (1966). High tibial osteotomy for arthritis of th $\overrightarrow{9}$ knee. Journal of Bone and Joint Surgery, 48B, 389.

Harris, W. R., and Kostnik, J.P. (1970). High tibial osteotomy for osteoarthritis of the knee. Journal of Bone and Join Surgery, 52A, 330-336.

Huskisson, E. C., and Phillips, H. (1973). Controlled trial of double osteotomy for rheumatoid arthritis. Rheumatologo and Rehabilitation, 12, 214-218.

Insall, J., Shoji, H., and Mayer, V. (1974). High tibiå osteotomy - a five year evaluation. Journal of Bone and Joint Surgery, 56A, 1397-1405.

Jackson, J. P. (1958). Osteotomy for osteoarthritis of the knee. Journal of Bone and Joint Surgery, 40B, 826.

Jackson, J. P., Waugh, W. and Green, J. P. (1969). High. tibial osteotomy for osteoarthritis of the knee. Journal oD Bone and Joint Surgery, 51 B, 88-94.

Jackson, J. P., and Waugh, W. (1974). The technique and complications of upper tibial osteotomy-a review of 226 operations. Journal of Bone and Joint Surgery, 56B. 236-245.

Shoji, H., and Insall, J. (1973). High tibial osteotomy fơ osteoarthritis of the knee with valgus deformity. Journal of Bone and Joint Surgery, 55A, 963-973.

Torgerson, W. R. (1965). Tibial osteotomy in the treatment of osteoarthritis of the knee. Surgical Clinics of Nort America, 45, 779-785.

Trueta, J. (1962). In discussion to Veenemans, C. J. (1962).

Trueta, J. (1963). Tibial osteotomy for osteoarthrosis of the knee. Journal of Bone and Joint Surgery, 45B, 618.

Veenemans, C. J. (1962). Tibial osteotomy for osteoarthrosifs of the knee. Journal of Bone and Joint Surgery, 44B, 95尺

Wardle, E. N. (1964). Osteotomy of the tibia and fibula id the treatment of chronic osteoarthritis of the knee Postgraduate Medical Journal, 40, 536-542.

Wiley, A. M. (1967). Reconstruction of the osteoarthritic knee by high tibial osteotomy and joint clearance. Canadian Journal of Surgery, 10, 28-35. 